\title{
High-Efficiency LED Driver Without Electrolytic Capacitor for Street Lighting
}

\author{
Manuel Arias, Member, IEEE, Diego G. Lamar, Member, IEEE, Javier Sebastián, Senior Member, IEEE, \\ Didier Balocco, and Almadidi Aguissa Diallo
}

\begin{abstract}
High-brightness light-emitting diodes (LEDs) are considered as a remarkable lighting device due to their high reliability, chromatic variety, and increasing efficiency. As a consequence, a high number of solutions for supplying LED strings are coming out. One-stage solutions are cost effective, but their efficiency is low as they have to fulfill several purposes with only one converter: power factor correction (PFC), galvanic isolation (in some cases), and current regulation. Two-stage and three-stage solutions have higher efficiency as each stage is optimized for just one or two tasks, and they are the preferred option when supplying several strings at the same time. Nevertheless, due to their higher cost in comparison to one-stage solutions, they are used when high efficiency, high performance, and the possibility of supplying several strings are the main concerns. In addition, they are also used when high reliability is needed and electrolytic capacitors cannot be used. In this paper, a three-stage solution and its complete design guideline for LED-based applications is proposed. PFC is achieved by a boost converter, while the galvanic isolation is provided by an electronic transformer (second stage). The third stages (one for each LED string) are designed following the two-input buck schematic, but taking advantage of the load characteristics (i.e., the high value of the LED string knee voltage, approximately equal to half the string nominal voltage). Moreover, a variation of the analog driving technique is also proposed. Experimental results obtained with a $160-\mathrm{W}$ prototype show an efficiency as high as $93 \%$ for the whole topology and $95 \%$ for the cascade connection of the second and third stages.
\end{abstract}

Index Terms-AC-DC power converters, electronic transformer, high efficiency, light-emitting diode (LED) lighting, threestage topology, two-input buck.

\section{INTRODUCTION}

$\mathbf{H}$ IGH-BRIGHTNESS light-emitting diodes (HB-LEDs) are considered the future trend in lighting as their efficacy converting energy into light is increasing and their reliability is very high [1]. Nevertheless, it is necessary to develop converters

Manuscript received January 11, 2012; revised March 7, 2012; accepted April 17, 2012. Date of publication November 15, 2012; date of current version January 16, 2013. Paper 2012-IPCC-031.R1, presented at the 2012 IEEE Applied Power Electronics Conference and Exposition, Orlando, FL, February 5-9, and approved for publication in the IEEE TRANSACTIONS ON INDUSTRY APPLICATIONS by the Industrial Power Converter Committee of the IEEE Industry Applications Society. This work was supported by AEG Power Solutions B.V. under Project FUO-EM-002-10 and by the Spanish Government under Projects CONSOLIDER MICINN-10-CSD2009-00046 and MICINN-10-DPI2010-21110-C02-01. A patent is pending.

M. Arias, D. G. Lamar, and J. Sebastián are with the Electronic Power Supply Systems Group, Universidad de Oviedo, 33204 Gijón, Spain (e-mail: ariasmanuel@uniovi.es; gonzalezdiego@uniovi.es; sebas@uniovi.es).

D. Balocco and A. A. Diallo are with AEG Power Solutions B.V., 22301 Lannion, France (e-mail: DIDIER.BALOCCO@aegps.com; ALMADIDI. DIALLO@aegps.com)

Color versions of one or more of the figures in this paper are available online at http://ieeexplore.ieee.org.

Digital Object Identifier 10.1109/TIA.2012.2227644

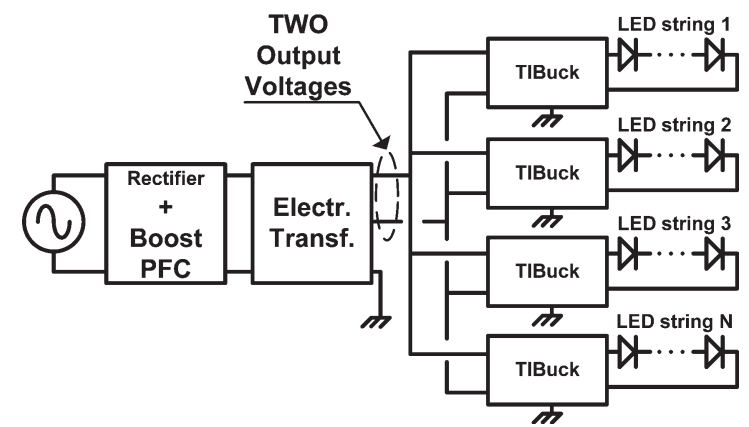

Fig. 1. Basic schematic of the proposed topology.

that perfectly fit the characteristics of these devices and make the best of them. According to this, two driving techniques have been proposed in the literature, and topologies without electrolytic capacitor (high reliability) [2] and high efficiency have been developed.

Regarding the converters for supplying the LED strings, it should be mentioned that LEDs need to be supplied with a dc current, and, consequently, ac-dc and dc-dc converters have been proposed in literature. According to the number of stages, these converters can be classified in one, two, or threestage solutions. The main characteristics that the converters for LED-based lighting need to ensure are high reliability, high efficiency, and, in some cases, galvanic isolation. In addition, the power factor correction (PFC) is mandatory in all ac-dc topologies connected to the line in order to comply with EN 61000-3-2 Class C regulation [3]-[5].

Normally, two-stage [6]-[8] and three-stage [9] solutions are the preferred option when reliability and efficiency are more important concerns than cost (per unit) [10]. LED-based street lighting is an application in which the cost of the LED driver is less important than its efficiency due to the amount of energy consumed by street lighting every day. Moreover, the maintenance and replacement cost of street-lighting drivers is considerably higher than in home applications [11]-[14]. Hence, reliability is also a key issue as it represents a considerable saving. Therefore, two-stage or three-stage topologies are the preferred option for LED-based street lighting.

In this paper, a high-efficiency high-reliability three-stage topology is proposed for LED-based street lighting application (see Fig. 1). The first stage is a boost converter [7]-[9]. This topology has proven to be a perfect option for doing PFC in this kind of application. As it is designed without electrolytic capacitor, its output voltage ripple cannot be neglected and will strongly influence the design of the second and third stages. The second stage is a two-output electronic transformer (ET) 
optimized for providing galvanic isolation at very high efficiency. Nevertheless, this topology is completely unregulated, and, as a consequence, the aforementioned low-frequency ripple affects its output voltages. The third stage is a topology based on the two-input buck (TIBuck) converter [15]-[18]. This third stage is in charge of eliminating the low-frequency ripple and independently adjusting the output current to the desired level in each LED string (i.e., no equalizing technique [19]-[21] is needed as each LED string has its own regulator [22]). The main advantage of this topology is that the stress on its components is considerably reduced, what has a high impact on its size and efficiency. This topology has already been proposed as post-regulator for LED-based applications [23], [24]. In [24], the TIBuck is used as an active equalizer (limited regulating capability) with very high efficiency, but without the possibility of providing full dimming on its own [20]. As it will be deeply explained, the key point of the TIBuck proposed in this paper is that it takes benefit from the high value of the LED string knee voltage [1]. This means that it can reach full dimming in LEDs although its output voltage range is limited. Finally, not only the first stage, but also the second and the third stages can be implemented without electrolytic capacitor. Hence, the proposed topology has a very high reliability.

As the final application is street lighting, some additional details should be considered. Wavelength (color) quality is less important than other issues like efficiency. In addition, the stress on the LEDs should be the lowest as a way of boosting the reliability. These points can be achieved by means of, among other things, the use of amplitude-mode driving technique as it has lower current stress on LEDs and semiconductors than the pulse width modulation (PWM) driving technique. Two other important aspects should be highlighted: the whole converter operates at constant frequency, and it provides galvanic isolation, which is mandatory in certain applications or for certain customers.

This paper is organized as follows. In Section II, the ET design is developed for this application, making some specific recommendations. Section III explains the new driving technique. The third stage is explained in Section IV. In Section V, a guideline on how to design stages two and three is provided. In Section VI, the experimental results are shown, and, finally, in Section VII, the conclusions will be presented.

\section{ET (Second Stage)}

Before explaining the second stage, it should be mentioned that the first stage is a boost converter operating in boundary conduction mode (BCM). This topology may reach an efficiency as high as $97 \%$ without electrolytic capacitor, and, as a consequence, it is widely used in two-stage and three-stage topologies for LED-based street lighting. The most relevant aspect is that, due to the absence of electrolytic capacitor, its output voltage has to be expressed as

$$
V_{\text {out_1 }}=V_{\text {out_1_nom }} \cdot\left(1+\frac{r_{v}}{2} \cdot \sin \left(2 \cdot \omega_{\text {in }} \cdot t\right)\right)
$$

where $\omega_{\text {in }}=2 \pi \cdot f_{\text {in }}, f_{\text {in }}$ being the line frequency, $V_{\text {out_1_nom }}$ is the nominal value of the first stage output voltage, and $r_{v}$ is the relative value of the peak-to-peak low-frequency ripple.

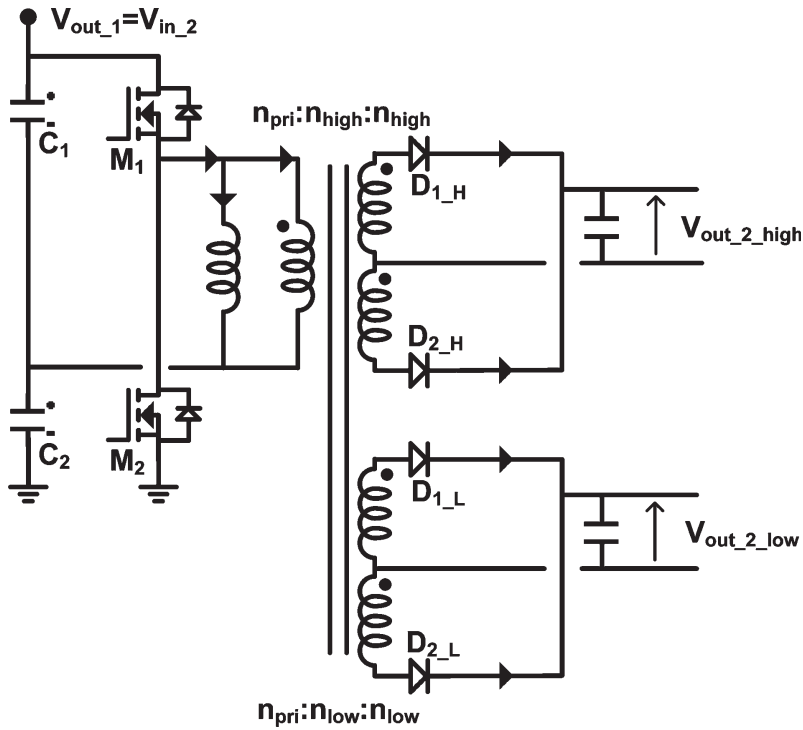

Fig. 2. Schematic of the electronic transformer.

This ripple will affect the second stage and the overall design of the proposed topology.

As has been said, galvanic isolation is a mandatory requirement. Hence, it is vital to find a topology capable of providing it with very high efficiency. If the second stage is also in charge of canceling the low-frequency ripple as in [9], then its output voltage has to be regulated, and the ET concept cannot be used. Nevertheless, as the third stages are regulated in order to control the LED string current, then they can also be in charge of canceling the low-frequency ripple. As a consequence, the only requirement applicable to the second stage is providing the galvanic isolation. This opens the possibility of using an ET, which is an unregulated topology with very high efficiency. The other option, using a galvanic-isolated topology for the third stages (no second stage needed), would lead to as many magnetic transformers as LED strings, which unnecessarily raises cost and size.

In this LED-based street lighting driver, the ET concept developed in [25] is applied to a two-output half-bridge topology (see Fig. 2). The main idea is operating the primary switches at constant switching frequency and with a fixed duty cycle of 50\% (disregarding the dead times necessary for achieving zero voltage switching (ZVS) and for avoiding overlapping). As a consequence, the dc conversion ratios (gains) between the input and the two outputs are fixed (i.e., it has no regulating capabilities). This means that any variation in the input voltage will affect the output voltages. The reason for having two outputs will be explained in Sections III and IV. Therefore, the two output voltages of the ET can be expressed as

$$
\begin{gathered}
V_{\text {out_2_high }}=G_{\text {high }} \cdot V_{\text {out_1_nom }} \cdot\left(1+\frac{r_{v}}{2} \cdot \sin \left(2 \cdot \omega_{\text {in }} \cdot t\right)\right) \\
V_{\text {out_2_low }}=G_{\text {low }} \cdot V_{\text {out_1_nom }} \cdot\left(1+\frac{r_{v}}{2} \cdot \sin \left(2 \cdot \omega_{\text {in }} \cdot t\right)\right)
\end{gathered}
$$

where $G_{\text {high }}$ and $G_{\text {low }}$ are the gains of the ET for both outputs and are fixed values determined by the "magnetic" transformer used in the topology. It should be noted that the term "magnetic" is going to be used in order to differentiate between the topology (ET) and the component ("magnetic" transformer). 


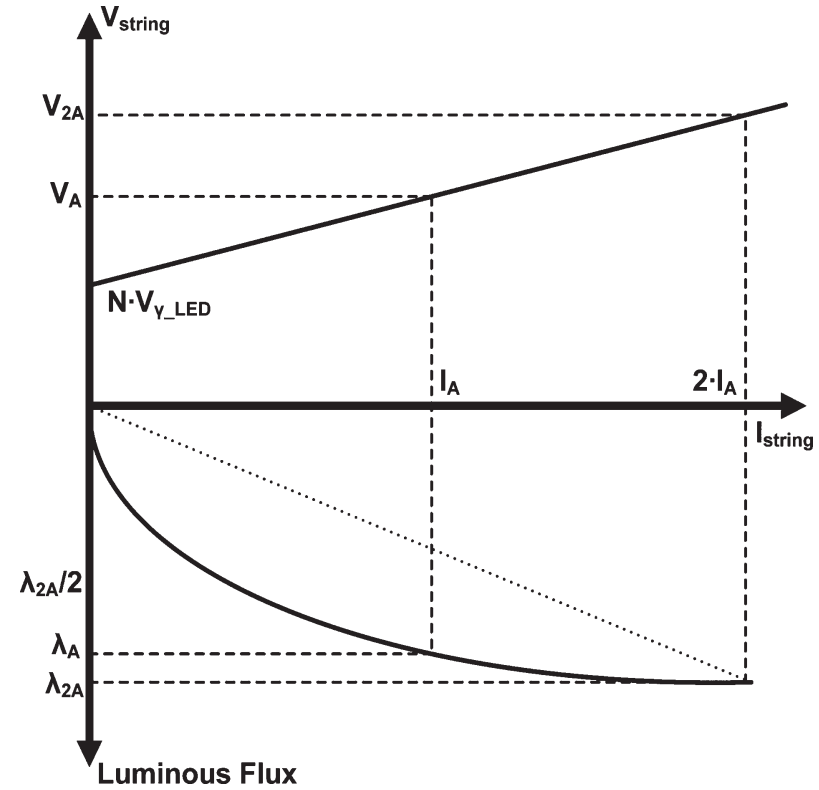

Fig. 3. Relation between input voltage, current, and luminous flux in a LED string. Saturation is enlarged for clarity reasons.

High efficiency is one of the objectives of this three-stage topology. Hence, ZVS and zero current switching (ZCS) are mandatory in this stage in order to reduce the losses. ZCS in diodes can be easily obtained by means of the resonance between the output capacitor and the leakage inductances of the "magnetic" transformer. It should be taken into account that this resonance is possible because there is no filter inductor in the outputs. In additions, the primary switches (MOSFETs) are quite close to ZCS. This is all perfectly explained in [25], where all the equations needed for calculating the output capacitor for a given leakage inductance, frequency, and dead time are provided. Nevertheless, these equations are thought for only one output. Hence, they need to be carefully used taking into account the worst possible load sharing between both outputs in order to always achieve ZCS.

\section{BRIEF DESCRIPTION OF THE PRoposed DRIVING TECHNIQUE FOR LEDS}

Normally, the converters in charge of regulating the dimming of the LED string current are always designed in such a way that they can vary their output voltage from zero to nominal value (i.e., buck topology). Nevertheless, if the special characteristics of LED strings are taken into account (i.e., the high value of the knee voltage and the dynamic resistance), it is possible to propose a new driving technique which opens a new field for novel topologies.

In Fig. 3, a relation between the voltage supplied to a string of N LEDs and the corresponding current is given. In addition, this current is related to the luminous flux of the string. As can be seen, LED strings emit an amount of light $\left(\lambda_{\mathrm{A}}\right)$ nearly proportional to the current driven by them $\left(I_{\mathrm{A}}\right)$. Nevertheless, they have an electrical model which involves an ideal diode, a dynamic resistance, and a voltage source, whose value is equal to the knee voltage of a single LED of the string $\left(V_{\gamma_{-} \text {LED }}\right)$ multiplied by the number of LEDs $(\mathrm{N})$. Hence, current driven by a LED is not directly proportional to the voltage applied to it $\left(V_{\mathrm{A}}\right)$. It is necessary to apply a threshold voltage (i.e., a voltage slightly higher than the previously mentioned voltage source) in order to have some current through the LED (i.e., some light emitted). Hence, for totally regulating the amount of light emitted by a LED string, it is not necessary to use topologies with the capability of reaching zero voltage at their output. In fact, it is possible to use converters (topologies) which have a minimum output voltage higher than zero, as far as this minimum voltage is lower than the equivalent knee voltage of the LED string $\left(N \cdot V_{\gamma \_L E D}\right)$.

This new approach to the driving technique (from this point, it will be defined as "total current dimming") allows the development of new topologies that sacrifice output voltage range but, as a counterpart, raise their efficiency and reduce the stress on their semiconductors. As has been mentioned, due to the knee voltage of their load (i.e., LED strings), these new topologies still have the possibility of totally turning off the strings (i.e., stopping the emission of light) even with a considerable voltage at their output.

Finally, it should be mentioned that this approach to the driving technique is compatible with both amplitude-mode and PWM-mode dimming. In addition, adding communication options, such as Digital Addressable Lighting Interface (DALI), to this driver is straightforward. It can be easily turned into a DALI Electronic Control Gear simply adding some additional components (essentially, a microcontroller or equivalent). This modification would provide the proposed driver with the possibility of storing the power-on level, 16 preset scene levels, etc.

\section{TIBUCK CONVERTERS (THIRD STAGES)}

\section{A. Brief Description and Steady-State Analysis}

The third stages are responsible for eliminating the voltage ripple coming from the ET outputs (due to the first stage) [26][28] and adjusting each LED string current to the desired level.

As can be seen in Fig. 4, the TIBucks are to some extent similar to the conventional buck converters used to drive LEDs, but the anode of the diode is connected to a voltage $V_{3}$ low lower than $V_{3}$ high, which would be the input voltage of a conventional buck. Hence, for a given duty cycle, the input voltage of the output filter $V_{f}$ is similar to the one shown in Fig. 4. Applying the volt -second balance in the output inductor results in

$$
\left(V_{3 \_ \text {high }}-V_{\text {out_3 }}\right) \cdot D+\left(V_{3 \_ \text {low }}-V_{\text {out_3 }}\right) \cdot(1-D)=0
$$

where $\mathrm{D}$ is the duty cycle of the MOSFET and $V_{3}$ high and $V_{3}$ low are the input voltages of the TIBuck. From (4), the output voltage equation can be obtained

$$
V_{\text {out_3 }}=V_{3 \_ \text {high }} \cdot D+V_{3 \_ \text {low }} \cdot(1-D) \text {. }
$$

Compared to a conventional buck, the main advantages of this topology are mainly two. First, as can be seen in Fig. 4, the input voltage of the filter is easier to filter, and, consequently, its size is smaller, which allows us to implement it without electrolytic capacitor. Second, the voltages withstood by the MOSFET and the diode are considerably lower than in a traditional buck

$$
V_{\mathrm{S} \_ \text {max }}=V_{\mathrm{D} \_ \text {max }}=V_{3 \_ \text {high }}-V_{3 \_ \text {low }}
$$



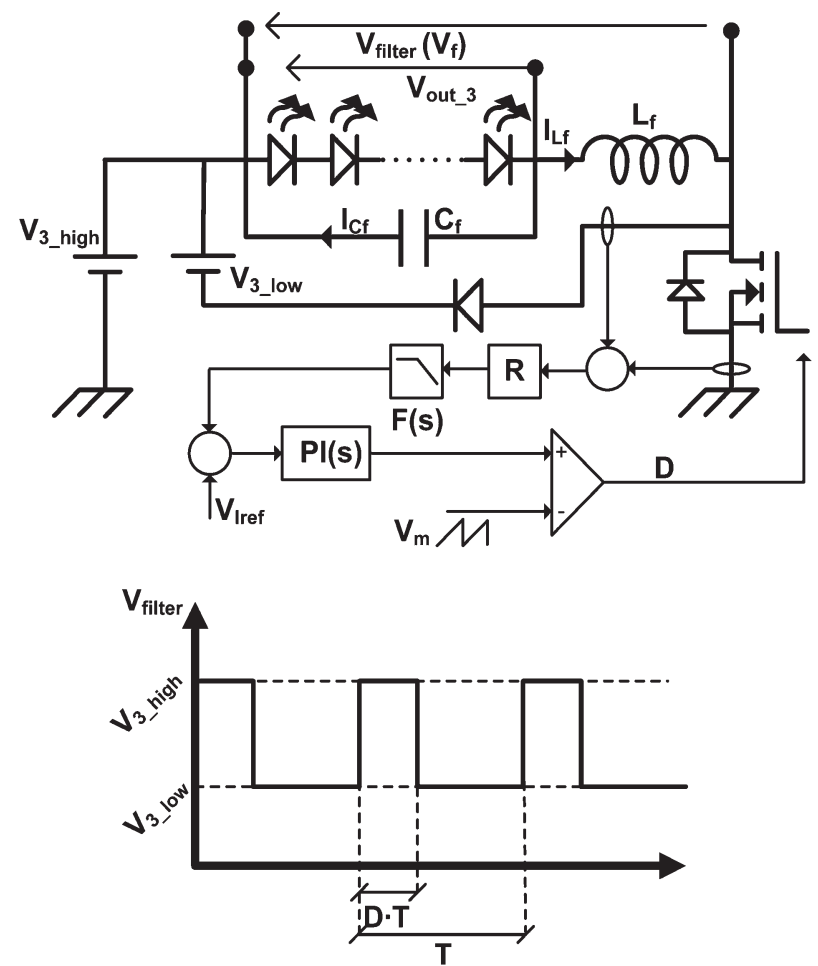

Fig. 4. Schematic of the proposed TIBuck converter proposed for driving each LED string.

where $V_{\mathrm{S}_{-} \max }$ and $V_{\mathrm{D}_{-} \max }$ are the maximum voltages withstood by the MOSFET and the diode of the TIBuck, respectively. As can be seen, they can be rated for a lower voltage (in the buck converter, they should be rated for a voltage equal to $V_{3 \_ \text {high }}$, and, consequently, this fact increases the efficiency of the proposed converter. In addition, the MOSFET is referred to ground for this application, so its gate driver is easy to implement.

It should be noted that the energy processed by a buck converter controlling a LED string comes from the single input port of this converter, while the processed energy in the case of each TIBuck comes from its two inputs. However, the total energy in both cases is similar (actually, it is lower in the case of the TIBuck due to its improved efficiency and better processing of energy).

The main disadvantage of the TIBuck is that its minimum output voltage is limited to the value of $V_{3} \_$low. Hence, it is not possible to reach $0 \mathrm{~V}$ or any voltage lower than $V_{3}$ low at the output. Nevertheless, as has been explained in Section III, the special characteristics of the load allow the use of this topology without losing the possibility of reaching full dimming in the strings. The only requirement is that $V_{3 \_ \text {low }}$ has to be chosen in such a way that, for the minimum duty cycle of the TIBuck, a voltage slightly lower than $N \cdot V_{\gamma_{-} L E D}$ is achieved. This is one of the key points of the design procedure and will be deeply explained in the following section. A minimum output voltage higher than zero also presents a problem when the output is short-circuited. Nevertheless, in this particular case, it is possible to solve the problem by turning off the second stage (i.e., the ET) when this situation is detected. The circuit for detecting this situation in the third stages and sending the turning-off signal to the ET is quite simple. Another possible solution is implementing fuses in the input or output of each TIBuck. In this way, it is not necessary to turn off the ET, and the strings which are not short-circuited still can be used. If part of a string is short-circuited, total dimming of that string current is lost, and turning off the lamp (i.e., all strings) can only be achieved by turning off the second stage then. In this case, using fuses is the most recommendable solution. Nevertheless, it is possible to reduce the impact of partially short-circuited strings if a security factor is added when calculating the low input voltage of the TIBucks. In this way, it is possible to totally control the string current even when some of its LEDs are short-circuited. Obviously, a tradeoff between this security factor and efficiency has to be met as the lower $V_{3}$ low, the lower the efficiency.

As has been said, it should be taken into account that, the closer $V_{3 \_ \text {high }}$ and $V_{3 \_ \text {low }}$, the lower will be the voltages that the semiconductors (MOSFET and diode) will have to withstand. In addition, the size of the magnetic component will also be lower. Taking into account the high value of the LED-string knee voltage (around half the nominal voltage), the improvement in efficiency and size is considerably high when using the TIBuck for LED-based applications.

\section{B. Small-Signal Analysis: Stability and Audiosusceptibility}

As has been mentioned, the third stages have to, not only regulate the current provided to the LED string, but also cancel the low-frequency ripple that affects the output voltages of the first and the second stages. This cancellation is achieved by means of the closed-loop controller and, consequently, the audiosusceptibility analysis is a key point in the design of the TIBuck. The linear equations that define the behavior of this third stage topology are

$$
\begin{aligned}
V_{f} & =v_{3 \_ \text {high }} \cdot d+v_{3 \_l o w} \cdot(1-d) \\
V_{f}-v_{\text {out_3 }} & =L_{f} \cdot \frac{d i_{\mathrm{Lf}}}{d t} \\
i_{\mathrm{Cf}} & =C_{f} \cdot \frac{d v_{\text {out_3 }}}{d t} \\
v_{\text {out_3 }}-N \cdot v_{\gamma_{\mathrm{LED}}} & =N \cdot R_{\mathrm{LED}} \cdot i_{\text {out_3 }}
\end{aligned}
$$

where $V_{f}$ is the input voltage of the output filter, $I_{\mathrm{Cf}}$ is the current through the filter capacitor, and $I_{\mathrm{Lf}}$ is the inductor current (see Fig. 4). Perturbing these equations, we obtain

$$
\begin{aligned}
V_{f}= & v_{3 \_ \text {high }} \cdot D+v_{3 \_ \text {low }} \cdot(1-D) \\
& +d \cdot\left(V_{3 \_ \text {high }}-V_{3 \_ \text {low }}\right) \\
V_{f}-v_{\text {out_3 }}= & L_{f} \cdot s \cdot \hat{i}_{\mathrm{Lf}} \\
\hat{i}_{\mathrm{Cf}}= & C_{f} \cdot v_{\text {out_3 }} \\
v_{\text {out_3 }}= & N \cdot R_{\mathrm{LED}} \cdot \hat{i}_{\text {out_3 }}
\end{aligned}
$$

where quantities in capital letters correspond to the steadystate values of the quantities in lower-case letters. With these equations, the following transfer functions can be obtained:

$$
\begin{aligned}
G_{\text {iout_vf }}(s) & =\frac{\hat{i}_{\text {out_3 }}}{V_{f}} \\
& =\frac{1}{s^{2} \cdot L_{f} \cdot C_{f} \cdot N \cdot R_{\text {LED }}+s \cdot L_{f}+N \cdot R_{\text {LED }}} \\
G_{\text {iout_d }}(s) & =\frac{\hat{i}_{\text {out_3 }}}{d}=\left(V_{3 \_ \text {high }}-V_{3 \_l o w}\right) \cdot G_{\text {iout_vf }}(s) .
\end{aligned}
$$


Analyzing the control loop shown in Fig. 4, it can be seen that the inductor current is sensed by means of two current transformers (which merge their outputs) plus a low-pass filter. Hence,

$$
d=\frac{P I(s)}{V_{m}} \cdot\left(\hat{i}_{\mathrm{ref}}-R \cdot F(s) \cdot \hat{i}_{\mathrm{Lf}}\right)
$$

where $\mathrm{R}$ is the gain of the current transformers, $V_{m}$ the peak value of $V_{\mathrm{tr}}, \mathrm{PI}(\mathrm{s})$ is the regulator, and $\mathrm{F}(\mathrm{s})$ represents the lowpass filter at the output of the current transformers. Considering (16) and (17), the equation of the system in close loop can be expressed as (18), shown at the bottom of the page.

For analyzing the audiosusceptibility, (17) can be expressed as

$$
G_{\mathrm{d} \_ \text {ilf }}(s)=\frac{d}{\hat{i}_{\mathrm{Lf}}}=-\frac{P I(s) \cdot R \cdot F(s)}{V_{m}} .
$$

Considering that the second stage has a fixed gain for each of its outputs, (11) can be expressed as

$$
\begin{array}{r}
v_{f}=\left[G_{\text {high }} \cdot D+G_{\text {low }} \cdot(1-D)\right] \cdot v_{\text {out } \_1 \_ \text {nom }} \\
+d \cdot\left(V_{3 \_ \text {high }}-V_{3 \_ \text {low }}\right) .
\end{array}
$$

Therefore, the audiosusceptibility, defined as the output current (i.e., LED string current) divided by the input voltage of the second stage, is represented by (21), shown at the bottom of the page, which, as can be seen, is not adimensional $\left(\Omega^{-1}\right)$. With this equation, it is possible to design a suitable controller PI(s) that obtains a reasonable ripple in the output current for a given low-frequency ripple in the input voltage. Considering that a PI controller is enough for reaching stability, the only variables for adjusting the stability and the audiosusceptibility are two: the gain of the controller (its pole would be located at a very low frequency) and the pole defined by the filter at the output of the current transformers. In the next section, some tips will be provided regarding this point.

\section{Overall Design Guideline}

The design of the three-stage topology should begin with the third stages (TIBucks) but fixing the value of the first-stage output voltage and its ripple. A typical ripple value, taking into account that no electrolytic capacitor is going to be used, is about $10 \%$. The other values that should be known are given by the LED strings: number of LEDs per string $(\mathrm{N})$, knee voltage of each LED $\left(V_{\gamma}\right.$ LED $)$, its dynamic resistance $\left(R_{\mathrm{LED}}\right)$, and its nominal current $\left(I_{\text {nom }}\right)$ (or its nominal power $\left(P_{\text {nom }}\right)$ ).

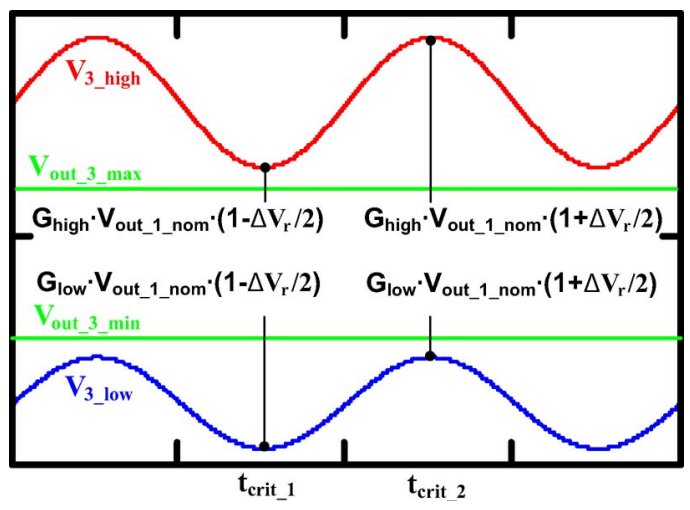

Fig. 5. Influence of the ripple in the second and third stages.

The maximum and minimum values of the output voltages of the TIBuck can be calculated as

$$
\begin{aligned}
& V_{\text {out_3_max }}=N \cdot V_{\gamma_{\mathrm{LED}}}+N \cdot \underline{R}_{\mathrm{LED}} \cdot I_{\mathrm{nom}}=N \cdot \frac{P_{\mathrm{nom}}}{I_{\mathrm{nom}}} \\
& V_{\text {out_3_min }}=N \cdot V_{\gamma \_\mathrm{LED}} .
\end{aligned}
$$

Considering (5) and defining $D_{\max }$ and $D_{\min }$ as the maximum and minimum duty cycles attainable by the control circuits of the TIBucks, we have

$$
\begin{aligned}
& V_{\text {out_3_max }}=D_{\max } \cdot V_{3 \_ \text {high }}+\left(1-D_{\max }\right) \cdot V_{3 \_ \text {low }} \\
& V_{\text {out_3_min }}=D_{\min } \cdot V_{3 \_ \text {high }}+\left(1-D_{\min }\right) \cdot V_{3 \_ \text {low }} .
\end{aligned}
$$

As has been explained, the ET has no regulating capability. Hence, its output voltages (i.e., the input voltages of the TIBucks) will have a considerable ripple (equal, in relative value, to the Boost output voltage ripple). As can be seen in Fig. 5, there are two critical situations:

- Trying to obtain the maximum output voltage $\left(V_{\text {out_3_max }}\right)$ when the input voltages are the lowest due to the ripple.

- Trying to obtain the minimum output voltage $\left(V_{\text {out_3_min }}\right)$ when the input voltages are the highest due to the ripple.

Hence, taking into account (2), (3), (24) and (25), we obtain

$$
\begin{aligned}
V_{\text {out_3 max }}= & {\left[D_{\max } \cdot G_{\text {high }}+\left(1-D_{\max }\right) \cdot G_{\text {low }}\right] } \\
& \cdot V_{\text {out_1_nom }} \cdot\left(1-\frac{r_{v}}{2}\right) \\
V_{\text {out_3 min }}= & {\left[D_{\text {min }} \cdot G_{\text {high }}+\left(1-D_{\text {min }}\right) \cdot G_{\text {low }}\right] } \\
& \cdot V_{\text {out_1_nom }} \cdot\left(1-\frac{r_{v}}{2}\right) .
\end{aligned}
$$

This system of two equations and two unknown variables can be easily solved, obtaining the values of $G_{\text {high }}$ and $G_{\text {low }}$.

$$
\frac{\hat{i}_{\text {out_3 }}}{\hat{i}_{\text {ref }}}=\frac{\frac{P I(s)}{s^{2} \cdot L_{f} \cdot C_{f} \cdot N \cdot R_{\mathrm{LED}}+s \cdot L_{f}+N \cdot R_{\mathrm{LED}}} \cdot \frac{V_{3} \_ \text {high }-V_{3} \_ \text {low }}{V_{m}}}{1+\frac{V_{3 \_ \text {high }}-V_{3} \_ \text {low }}{V_{m}} \cdot F(s) \cdot R \cdot P I(s) \cdot \frac{\left(1+s \cdot C_{f} \cdot N \cdot R_{\mathrm{LED}}\right)}{\left(s^{2} \cdot L_{f} \cdot C_{f} \cdot N \cdot R_{\mathrm{LED}}+s \cdot L_{f}+N \cdot R_{\mathrm{LED}}\right)}}
$$

$$
\frac{\hat{i}_{\text {out_3 }}}{v_{\text {out_1_nom }}}=\frac{G_{\text {iout_vf }}(s)}{\left[1-G_{\text {iout_vf }}(s) \cdot G_{\text {d_ilf }}(s) \cdot\left(v_{3 \_ \text {high }}-V_{3 \_l o w}\right) \cdot\left(1+s \cdot C_{f} \cdot N \cdot R_{\text {LED }}\right)\right]} \cdot\left(G_{\text {high }} \cdot D+G_{\text {low }} \cdot(1-D)\right)
$$


Once the two gains of the ET have been obtained, its "magnetic" transformer can be designed. First of all, the turns ratios of this transformer ( $R t_{\text {high }}$ and $\left.R t_{\text {low }}\right)$ can be defined as

$$
\begin{aligned}
R t_{\mathrm{high}} & =\frac{n_{\mathrm{high}}}{n_{\mathrm{pri}}} \\
R t_{\mathrm{low}} & =\frac{n_{\mathrm{low}}}{n_{\mathrm{pri}}}
\end{aligned}
$$

where $n_{\text {pri }}$ is the number of turns of the primary winding and $n_{\text {high }}$ and $n_{\text {low }}$ are the number of turns of the secondary windings of each output (see Fig. 2). Considering that the ET operates with a fixed duty cycle of about 0.5 , the relation between its input and its two output voltages can be expressed as

$$
\begin{aligned}
V_{\text {out_2_high }} & =\frac{1}{2} \cdot R t_{\text {high }} \cdot V_{\text {in_2 }} \\
V_{\text {out_2_low }} & =\frac{1}{2} \cdot R t_{\text {low }} \cdot V_{\text {in_2 }}
\end{aligned}
$$

so

$$
\begin{aligned}
R t_{\text {high }} & =2 \cdot G_{\text {high }} \\
R t_{\text {low }} & =2 \cdot G_{\text {low }} .
\end{aligned}
$$

In (30) and (31), the dead times required for achieving ZVS have not been considered. Although their influence is low, a conservative calculation would imply a total dead time of $10 \%$, which would lead to a duty cycle of $45 \%$.

Although conventional transformers have no airgap in the magnetic core, in this design, it is mandatory to reach ZVS in the primary switches. As has been explained, due to the low value of the input current of the ET, the best option is adding the necessary airgap that assures a high-enough magnetizing current.

The next element to be designed is the output capacitor of the ET. Its value is defined by the equations presented in [25]. In this case, it is quite difficult to estimate the leakage inductance due to the complex design of the transformer (one input winding and two center-tapped output windings). Hence, the best option is making the "magnetic" transformer with the parameters obtained during the theoretical design and, once this is accomplished, measuring the leakage inductances in each winding. With these values, it is possible to calculate the capacitor of each output of the ET in order to achieve ZCS in secondary switches and nearly ZCS in the primary ones.

Finally, the component selection is immediate. The MOSFET and diode of the TIBuck are going to withstand a voltage equal to the difference of both input voltages (see (6)). The average currents passing through the MOSFET and the diode, in the worst case, are equal to the nominal current demanded by one LED string multiplied by $D_{\max }$ and $\left(1-D_{\min }\right)$, respectively

$$
\begin{aligned}
& I_{\mathrm{S} \_ \text {max }}=I_{\text {nom }} \cdot D_{\max } \\
& I_{D_{\text {_ }} \text { max }}=I_{\text {nom }} \cdot\left(1-D_{\min }\right) .
\end{aligned}
$$

In the case of the ET, the MOSFETs are going to withstand a voltage equal to the maximum value of the input voltage of the ET obtained from (1)

$$
V_{\text {MOSFET_ET }}=V_{\text {out_1_nom }} \cdot\left(1+\frac{r_{v}}{2}\right) .
$$

The output diodes rating voltage should be

$$
\begin{aligned}
V_{\text {diode_high_ET }} & =V_{\text {out_1_nom }} \cdot\left(1+\frac{r_{v}}{2}\right) \cdot R t_{\text {high }} \\
V_{\text {diode_low_ET }} & =V_{\text {out_1_nom }} \cdot\left(1+\frac{r_{v}}{2}\right) \cdot R t_{\text {low }} .
\end{aligned}
$$

The current should be calculated taking into account that this stage is common to all the strings. Hence, considering that the duty cycle is fixed and that the worst situation corresponds to all the current drained from only one of the two outputs (the other would be driving no current), the average values of semiconductor currents are

$$
\begin{aligned}
\overline{I_{\mathrm{MOSFET} \_\mathrm{ET}}} & =\frac{N \cdot I_{\mathrm{nom}}}{2} \cdot R t_{\mathrm{high}} \\
\overline{I_{\text {diode_ET }}} & =\frac{N \cdot I_{\mathrm{nom}}}{2} .
\end{aligned}
$$

Finally, the design of the third stages controllers needs to be made. First of all, it should be taken into account that the pole of the filter of the current transformers should be located at a frequency higher than that of the low-frequency ripple that needs to be removed. Hence, this pole has no influence in the audiosusceptibility at around $100 \mathrm{~Hz}$. In addition, the zero defined by $N \cdot R_{\mathrm{LED}}$ and $C_{f}$ is considerably higher than this frequency. Therefore, it has no influence in the audiosusceptibility at that frequency either. Considering this, the design procedure should be as follows:

- Adjusting the gain of the PI controller so that a valid audiosusceptibility is reached at $100 \mathrm{~Hz}$. The higher the gain, the lower the audiosusceptibility.

- Placing the pole of the current transformers filter at a frequency that provides a valid phase margin in the stability analysis.

- If it is not possible to obtain a stable system, the gain should be reduced until stability is reached. Obviously, adding poles and zeros to the controller may help in reaching stability without reducing the gain (i.e., without affecting audiosusceptibility at $100 \mathrm{~Hz}$ ). Nevertheless, a PI controller is normally enough.

\section{EXPERIMENTAL RESULTS}

A 160-W prototype has been built (see Fig. 6). Four TIBucks (one for each LED string) have been connected at the outputs of the ET. Hence, the prototype has the capability of independently driving four LED strings. The LEDs used for the lamp are W4218T2-SW from Seoul Semiconductors (distributed by Avnet). They belong to the Z-Power LED family. Their nominal forward current is $0.350 \mathrm{~A}$, and their nominal forward voltage is $3.25 \mathrm{~V}$ (the nominal power of each LED is $1.14 \mathrm{~W}$ ). In each string, 35 LEDs are connected in series. Therefore, the nominal output voltage of each string is around $115 \mathrm{~V}(35 \times 3.25 \mathrm{~V})$, and its nominal power is around $40 \mathrm{~W}(115 \mathrm{~V} \times 0.350 \mathrm{~A})$. The total power demanded by the lamp is $160 \mathrm{~W}$.

Regarding the boost converter (first stage), it operates in BCM in order to reduce switching losses. The output capacitance $(30 \mu \mathrm{F})$ is obtained by means of metallized polypropylene film capacitors (MKP) from EPCOS high density series [29]. In the prototype shown in Fig. 6, three 10- $\mu$ F capacitors rated for 


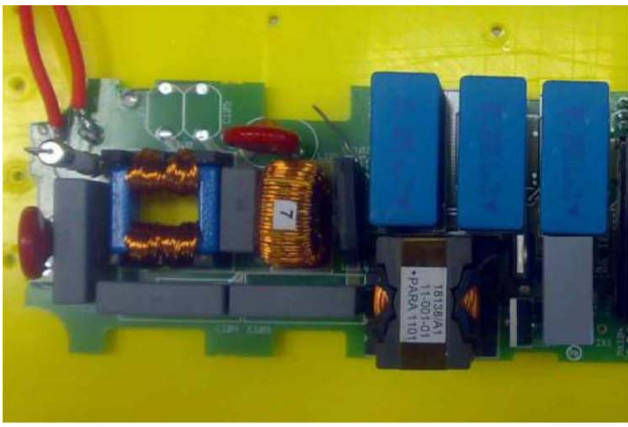

(a)

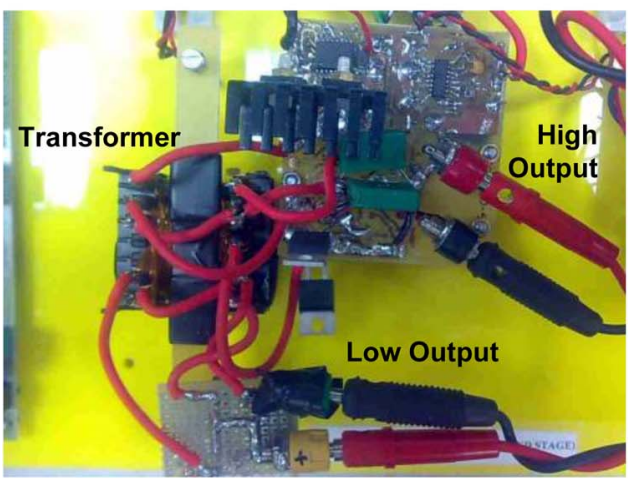

(b)

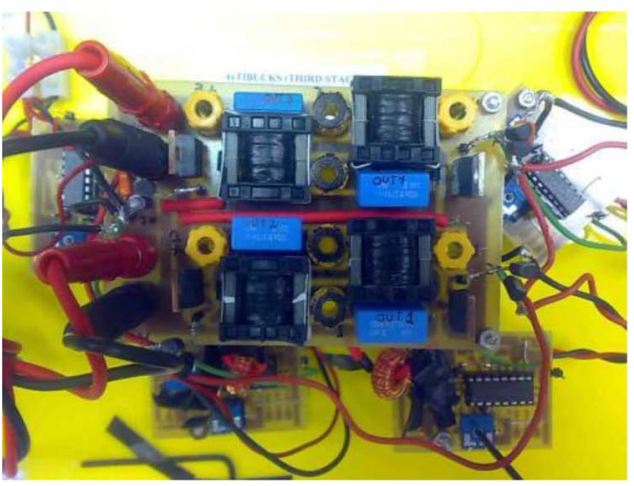

(c)

Fig. 6. (a) Prototype of the PFC Boost; (b) prototype of the 160-W ET; (c) prototype of the four 40-W TIBucks (one for each LED string).

$450 \mathrm{~V}$ are implemented. Another possibility would have been using a $30-\mu \mathrm{F}$ capacitor rated for $800 \mathrm{~V}$ if a higher security factor had been required regarding rated voltage.

The ET nominal output voltages are $80 \mathrm{~V}$ and $144 \mathrm{~V}$ (when the input voltage is $400 \mathrm{~V}$ ). They have been chosen taking into account (24) and (25) and the characteristics of the LED strings: they need $130 \mathrm{~V}$ to fully turn on and around $90 \mathrm{~V}$ to totally turn off. As can be seen, a 10-V security factor has been added in both output voltages in order to deal with the tolerance in the knee voltage of the LEDs and in their dynamic resistance. In addition, this security factor also allows the driver to deal with partially short-circuited strings. The input voltage of the ET is intended to be $400 \mathrm{~V}$ with a maximum peak-to-peak ripple of $10 \%$. The control of both MOSFETs runs at $100 \mathrm{kHz}$. The transformer is an ETD-39 with 3F3 magnetic core and 0.8$\mathrm{mm}$ gap in order to achieve ZVS at any load range in primary switches. For achieving ZCS, the equations presented in [25] imply that a $200-n F$ output capacitor is necessary.

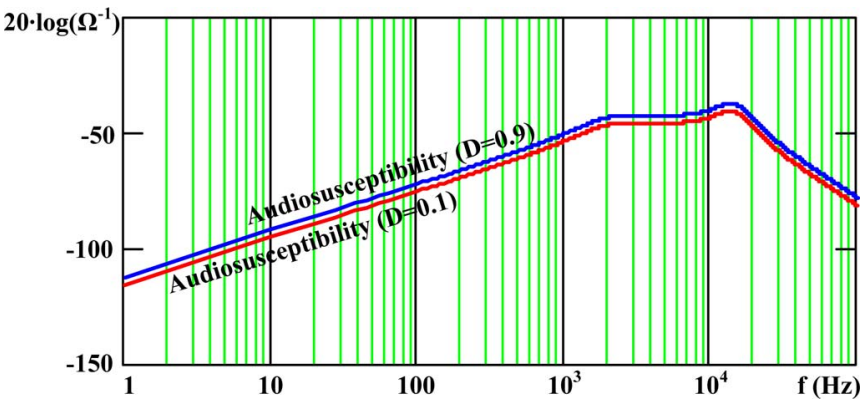

Fig. 7. Audiosusceptibility in closed loop for two different duty cycles.

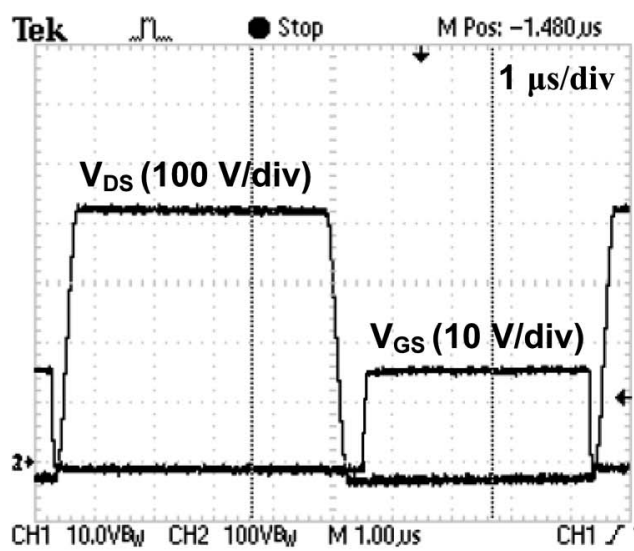

(a)

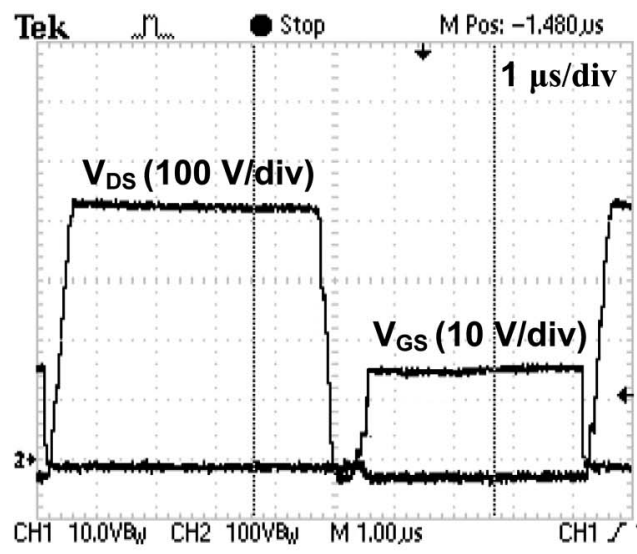

(b)

Fig. 8. ZVS in ET MOSFETs at (a) full load and (b) no load.

The four TIBucks are operated at $100 \mathrm{kHz}$. The output filter is implemented by means of an E20 core with a value of $0.350 \mathrm{mH}$ and an output capacitor of $150 \mathrm{nF}$. Due to the low voltage that both semiconductors are going to withstand $(144-80=64 \mathrm{~V})$, it is possible to use an IRF540 MOSFET and an 11DQ10 diode. These low-voltage-rated devices allow us to boost the efficiency of these converters. A very simple PI controller (with the pole located at very low frequency and a dc gain of about $80 \mathrm{~dB}$ ) is implemented in each TIBuck so that the current can be independently controlled while maintaining an audiosusceptibility as low as $0.0003 \Omega^{-1}$ at $100 \mathrm{~Hz}$ (see Fig. 7).

In Fig. 8, it can be seen how the primary switches (MOSFETs) of the ET achieve ZVS condition at full load and at no load. It has to be taken into account that the energy for doing so is stored in the leakage inductance and, therefore, is defined not 


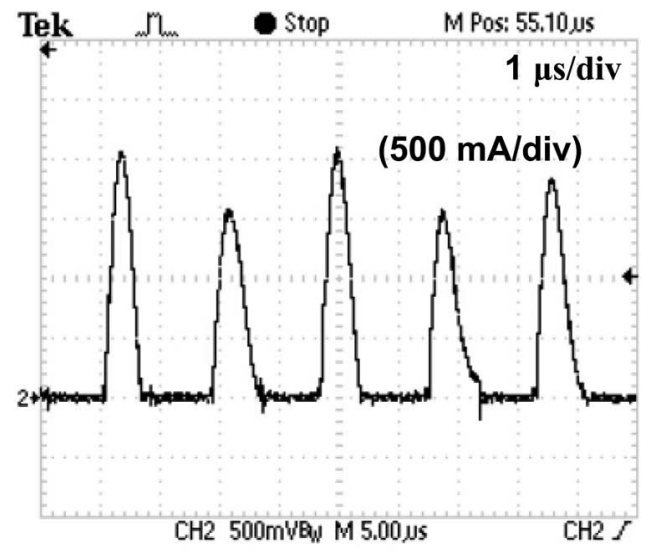

(a)

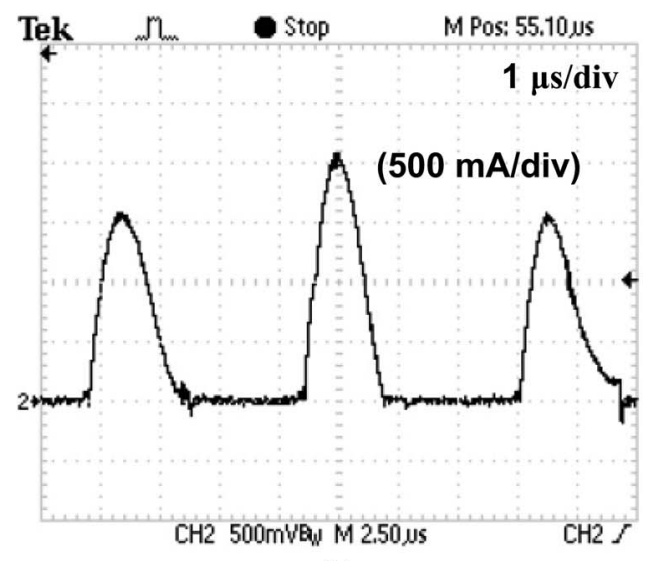

(b)

Fig. 9. (a) Current through one of the ET output diodes; (b) detail.

only by the load current, but also by the magnetizing current of the "magnetic" transformer. Hence, if some gap is added to the core of this transformer, it is possible to have enough energy stored in the leakage inductance for achieving ZVS even with an output current equal to zero. In this particular case, the gap was added because the amount of energy determined by the output current was not enough for achieving ZVS even at full load.

At this point, it may be interesting to determine whether it is better decreasing the magnetizing inductance of the transformer or not. If some airgap is added, the switching losses of the MOSFETs are going to be reduced (ZVS is achieved). Nevertheless, the current level through them, and through the transformer, is going to be increased, and so is going to be increased their conduction losses. For solving this, the same topology has been tested with and without airgap for the same testing condition and adjusting dead times for achieving ZVS (with airgap) or being the closest to it (with no airgap). The efficiency was $1 \%$ higher when adding some gap to reduce the switching losses than when minimizing it to reduce conduction losses.

Another characteristic of the ET is the possibility of achieving ZCS at secondary switches (diodes). In Fig. 9, this characteristic is shown for one of the diodes of the higher voltage output. Considering that the amount of current extracted from each output will strongly depend on the TIBucks operation, the best option is calculating the output capacitors of the ET considering that the nominal current is extracted from each

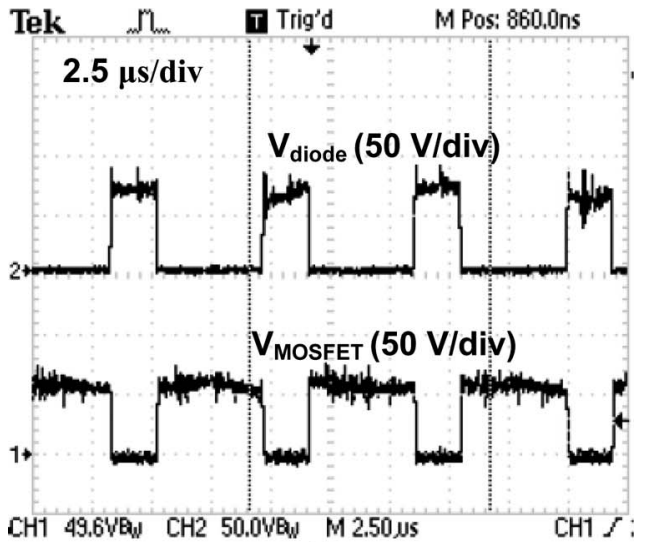

(a)

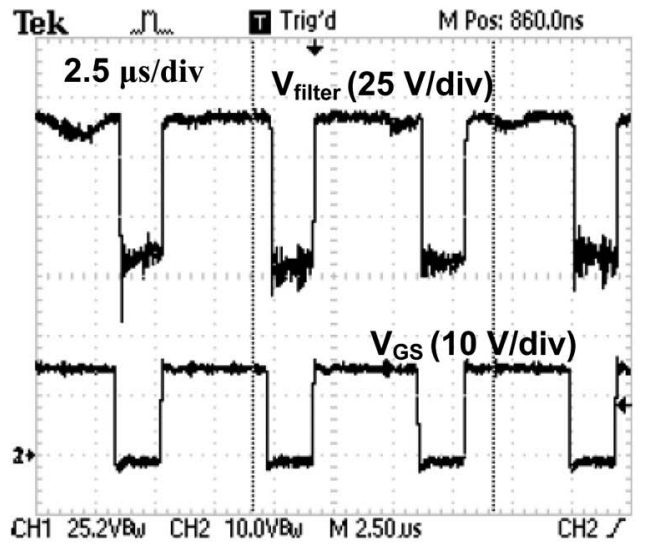

(b)

Fig. 10. (a) Voltage withstood by the TIBuck diode and MOSFET; (b) Gate signal of the MOSFET and filter input voltage.

output. In this way, diodes will always achieve ZCS in nominal conditions. The primary switches of the ET will also be closed to ZCS as the input current is the output one multiplied by the turns ratio, but adding the magnetizing current.

Regarding the TIBucks, Fig. 10 shows the voltage withstood by semiconductors and the input voltage of the output filter. As can be seen, the voltage withstood is equal to the difference between both input voltages. In this way, switching losses are considerably reduced. In addition, the necessary MOSFET and diode are going to be rated for a lower voltage, what means lower $R_{\mathrm{DSON}}, C_{\mathrm{oss}}$, voltage drop, and reverse recovery time, what also reduces losses. Analyzing Fig. 10(b), it can be seen that the output filter is going to be considerably smaller than in a conventional buck. This also leads to a reduction of the losses in the inductor.

In Fig. 11(a) and (b), the current through one of the strings is shown when second and third stages are connected in cascade and when the input voltage of the second stage has no ripple. As can be seen, there is no low-frequency ripple, and the highfrequency one is considerably reduced, particularly for an application like street-lighting. This high-frequency ripple could be even more reduced if the output capacitor of the third stage were increased (it has a value of $150 \mathrm{nF}$, so its capacitance could be easily increased without using electrolytic technology). In Fig. 11(c), the output current is shown when the input voltage of the second stage has a ripple of $10 \%$. In this system, the 


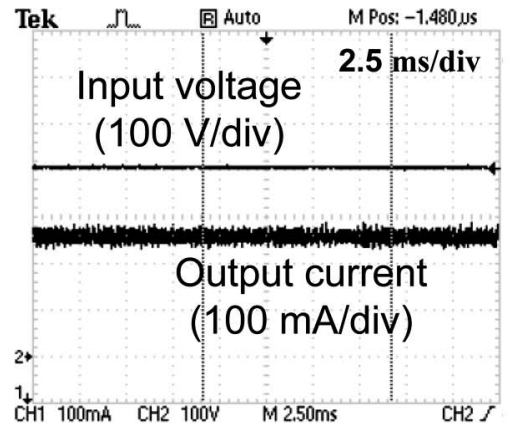

(a)

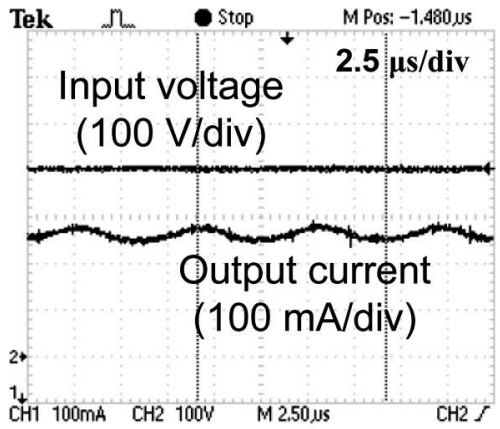

(b)

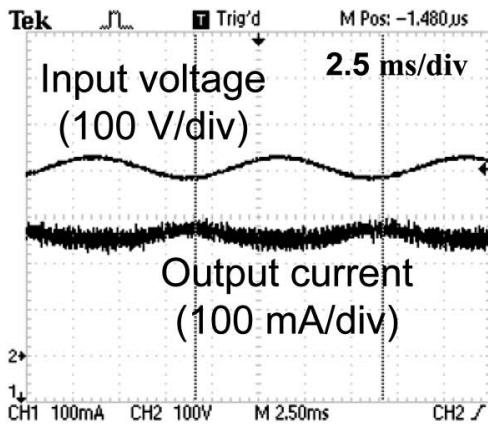

(c)

Fig. 11. ET input voltage and LED string current when (a) the input voltage has no low-frequency ripple; (b) detail when the input voltage has no low-frequency ripple; (c) the input voltage has a $45-\mathrm{V}_{\mathrm{pp}} 100-\mathrm{Hz}$ ripple.

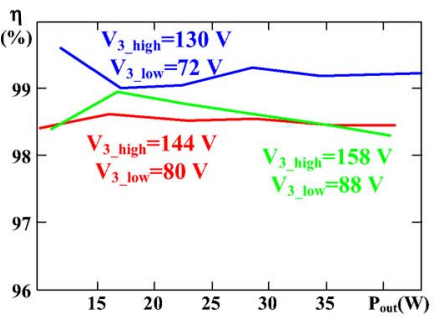

(a)

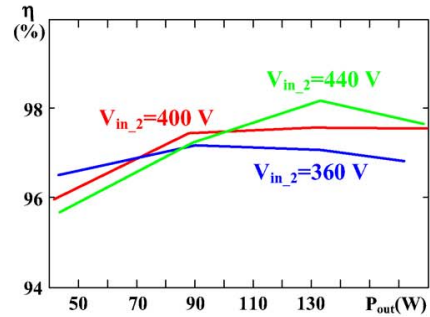

(b)

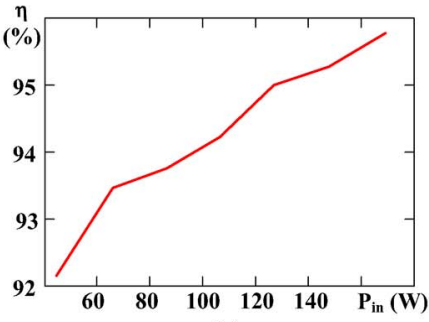

(c)

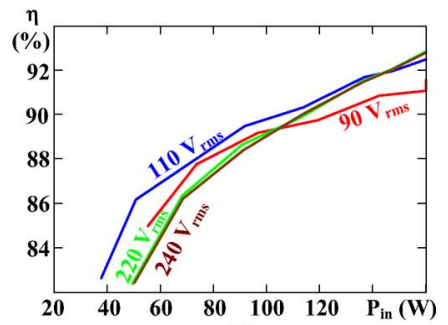

(d)

Fig. 12. (a) Efficiency of one of the TIBuck, (b) efficiency of the ET for different input voltages (control stage consumption is not included), (c) efficiency of the ET connected in cascade with the four modified bucks (control stage consumption is not included), and (d) efficiency of the three stages connected in cascade for different ac input voltages.

audiosusceptibility of the third stage has been defined as output current of the third stage divided by the input voltage of the second stage (see (21)). Taking into account the nominal values of these two variables, audiosusceptibility should be around $0.0003 \Omega^{-1}$ at twice the line frequency. In this way, a $10 \%$ ripple in the input voltage $\left(40 \mathrm{~V}_{\mathrm{pp}}\right)$ of the second stage will produce a $12-\mathrm{mA}_{\mathrm{pp}}$ low-frequency ripple in the output current of the TIBucks $\left(40 \mathrm{~V}_{\mathrm{pp}} \cdot 0.0003 \Omega^{-1}\right)$. This represents a $3.5 \%$ relative ripple in the output current $\left(12 \mathrm{~mA}_{\mathrm{pp}} / 350 \mathrm{~mA}\right)$, which is an excellent current ripple for street-lighting applications.

In Fig. 12, the efficiency of the second and the third stages is shown. Fig. 12(a) shows the efficiency of one of the TIBucks for different input voltages, which are defined by the maximum, nominal, and minimum input voltage of the ET. Fig. 12(b) shows the efficiency of the second stage for the maximum, nominal, and minimum input voltage. In both cases, the power consumption of the control stages is not included. This total power consumption is around $2 \mathrm{~W}$ for the four TIBucks plus the ET control stages. Hence, the efficiency of the second stage in cascade with the four third stages operating at full load is nearly $96.0 \%$ (see Fig. 12(c) for the whole-range efficiency). If the power consumption of the control stages is included, this efficiency is as high as $94.8 \%$. Considering that the second stage proposed in [9] has an efficiency as high as $97 \%$ and considering that the third stage of that topology (the conventional buck converters) has an efficiency as high as $94 \%$, the overall topology has an efficiency as high as $91 \%$. Hence, the topology proposed in this paper (ET plus the TIBucks) implies an improvement in efficiency of about $4-5 \%$.

Finally, as the BCM-PFC boost converter may reach an efficiency as high as $97 \%$, the overall efficiency of the proposed three-stage topology may be as high as 92-93\%. In Fig. 12(d), the efficiency of the three stages in cascade for different ac input voltages is shown.

\section{CONCLUSION}

HB-LEDs are gaining consideration in lighting due to their high efficacy when converting electrical energy into light. Nevertheless, this new technology needs power supplies different from the rest of lighting devices. The main issues when analyzing these new converters are high efficiency, high reliability, color quality, and total dimming. Nevertheless, there are applications in which some of this features are more important than others. In street lighting, light quality is less important than reliability and efficiency.

In this paper, a converter for LED-based street lighting application is presented. It is based in a three-stage topology. Each stage is designed for one specific task, in such a way that the overall efficiency is above $93 \%$. In addition, it is a solution without electrolytic capacitor; hence, it has high reliability.

The first stage of the proposed solution is the very-well known boost converter. Its main task is achieving PFC with high efficiency. The second stage is an ET. It provides two output voltages with a fixed gain (unregulated) between input and outputs. Its task is providing galvanic isolation. The third stage is a TIBuck. While the first two stages are common, each LED string is connected to a TIBuck. In this way, the current of each string can be regulated independently. In addition, the first stage has a considerable output voltage ripple due to the absence of electrolytic capacitors. As the second stage is unregulated, this ripple has to be cancelled by the third stage. 
The three stages have been implemented without electrolytic capacitor. The first stage has three $10-\mu \mathrm{F}$ MKP capacitors from EPCOS high density series. The ET has two MKP capacitors of $100 \mathrm{nF}$ in each output. Finally, each TIBuck (third stage) has a 150-nF output MKP capacitor.

The experimental results have been obtained with a prototype designed for $4 \times 40$-W LED strings achieving an efficiency at full load as high as $95 \%$ for the second and third stages in cascade. If the first stage is also considered, the proposed threestage LED driver may reach an efficiency as high as $93 \%$.

\section{REFERENCES}

[1] T. Siew-Chong, "General $n$ level driving approach for improving electrical-to-optical energy-conversion efficiency of fast-response saturable lighting devices," IEEE Trans. Ind. Electron., vol. 57, no. 4, pp. 1342-1353, Apr. 2010.

[2] G. Linlin, R. Xinbo, X. Ming, and Y. Kai, "Means of eliminating electrolytic capacitor in AC/DC power supplies for LED lightings," IEEE Trans. Power Electron., vol. 24, no. 5, pp. 1399-1408, May 2009.

[3] D. G. Lamar, J. Sebastián, A. Rodríguez, M. Rodríguez, and M. M. Hernando, "A very simple control strategy for power factor correctors driving high-brightness LEDs," IEEE Trans. Power Electron., vol. 24, no. 8, pp. 2032-2042, Aug. 2009.

[4] H. Ma, W. Yu, C. Zheng, J.-S. Lai, Q. Feng, and B.-Y. Chen, "A universalinput high-power-factor PFC pre-regulator without electrolytic capacitor for PWM dimming LED lighting application," in Proc. IEEE ECCE, 2011, pp. 2288-2295.

[5] H. Ma, W. Yu, Q. Feng, J.-S. Lai, and C. Zheng, "A novel SEPIC-derived PFC pre-regulator without electrolytic capacitor for PWM dimming LED lighting application based on valley fill circuit," in Proc. IEEE ECCE, 2011, pp. 2310-2317.

[6] K. I. Hwu, Y. T. Yau, and L. Li-Ling, "Powering LED using highefficiency SR flyback converter," IEEE Trans. Ind. Appl., vol. 47, no. 1, pp. 376-386, Jan./Feb. 2011.

[7] Q. Hu and R. Zane, "Off-line LED driver with bidirectional second stage for reducing energy storage," in Proc. IEEE ECCE, 2011, pp. 2302-2309.

[8] Q. Hu and R. Zane, "Minimizing required energy storage in off-line LED drivers based on series-input converter modules," IEEE Trans. Power Electron., vol. 26, no. 10, pp. 2887-2895, Oct. 2010.

[9] C. Spini, "48 V-130 W High Efficiency Converter with PFC for LED Sreet Lghting Aplications- European Version," STMicroelectronics, Geneva, Switzerland, AN3105 Appl. Note, 2010.

[10] D. Bailey, "An idea to simplify LED lighting purchase decisions," Bodo's Power System, Laboe, Germany, p. 18, 2011.

[11] J. J. Sammarco and T. Lutz, "Visual performance for incandescent and solid-state cap lamps in an underground mining environment," IEEE Trans. Ind. Appl., vol. 47, no. 5, pp. 2301-2306, Sep./Oct. 2011.

[12] X. Qu, S. C. Wong, and C. K. Tse, "Resonance-assisted buck converter for offline driving of power LED replacement lamps," IEEE Trans. Power Electron., vol. 26, no. 2, pp. 532-540, Feb. 2011.

[13] Y. Hu, L. Huber, and M. Jovanovic, "Single-stage, universal-input AC/DC LED driver with current-controlled variable PFC boost inductor," IEEE Trans. Power Electron., vol. 27, no. 3, pp. 1579-1588, Apr. 2012.

[14] P. Green, "Triac dimmers for LED lamps," BODO's Power Systems, Laboe, Germany, pp. 52-53, 2011.

[15] J. Sebastian, P. Villegas, F. Nubo, O. Garcia, and J. Arau, "Improving dynamic response of power factor preregulators by using two-input highefficient post-regulators," in Conf. Rec. 27th Annu. IEEE PESC, 1996, pp. 1818-1824.

[16] K. Gummi and M. Ferdowsi, "Double-input DC-DC power electronic converters for electric-drive vehicles-topology exploration and synthesis using a single-pole triple-throw switch," IEEE Trans. Ind. Electron., vol. 57, no. 2, pp. 617-623, Feb. 2010.

[17] J. Sebastian, P. J. Villegas, M. Hernando, F. Nuno, and F. FernandezLinera, "Average-current-mode control of two-input buck postregulators used in power-factor correctors," IEEE Trans. Ind. Electron., vol. 46, no. 3, pp. 569-576, Jun. 1999.

[18] J. Sebastian, P. J. Villegas, F. Nuno, and M. M. Hernando, "Highefficiency and wide-bandwidth performance obtainable from a two-input buck converter," IEEE Trans. Power Electron., vol. 13, no. 4, pp. 706-717, Jul. 1998.
[19] K. I. Hwu and Y. T. Yau, "Applying one-comparator counter-based sampling to current sharing control of multichannel LED strings," IEEE Trans. Ind. Appl., vol. 47, no. 6, pp. 2413-2421, Nov./Dec. 2011.

[20] H. Yuequan and M. M. Jovanovic, "LED driver with self-adaptive drive voltage," IEEE Trans. Power Electron., vol. 23, no. 6, pp. 3116-3125, Nov. 2008

[21] J. Wang, J. Zhang, X. Wu, Y. Shi, and Z. Qian, "A novel high efficiency and low-cost current balancing method for multi-LED driver," in Proc. IEEE ECCE, 2011, pp. 2296-2301.

[22] C. Van Dam, "Efficient power solutions for LED lighting installations," Bodo's Power Systems, Laboe, Germany, pp. 54-55, 2011.

[23] L. Wai-Keung, K. H. Loo, T. Siew-Chong, Y. M. Lai, and C. K. Tse, "Bilevel current driving technique for LEDs," IEEE Trans. Power Electron., vol. 24, no. 12, pp. 2920-2932, Dec. 2009.

[24] Y. Wensong, L. Jih-Sheng, M. Hongbo, and Z. Cong, "High-efficiency DC-DC converter with twin bus for dimmable LED lighting," IEEE Trans. Power Electron., vol. 26, no. 8, pp. 2095-2100, Aug. 2011.

[25] R. Yuancheng, X. Ming, S. Julu, and F. C. Lee, "A family of high power density unregulated bus converters," IEEE Trans. Power Electron., vol. 20 , no. 5, pp. 1045-1054, Sep. 2005.

[26] A. Wilkins, J. Veitch, and B. Lehman, "LED lighting flicker and potential health concerns: IEEE standard PAR1789 update," in Proc. IEEE ECCE, 2010, pp. 171-178.

[27] B. Lehman, A. Wilkins, S. Berman, M. Poplawski, and N. Johnson Miller, "Proposing measures of flicker in the low frequencies for lighting applications," in Proc. IEEE ECCE, 2011, pp. 2865-2872.

[28] B. Lehman and A. Shteynberg, "Professional Education Seminars: LED lighting: Trends, standards, optics and power electronics," in Proc. IEEE APEC, Fort Worth, TX, 2011.

[29] "Film Capacitors. Metallized Polypropylene Film Capacitors (MKP). DC Link High Density Series,” EPCOS, Munich, Germany, 2009.

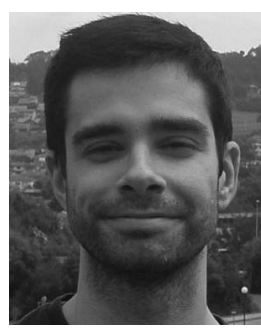

Manuel Arias (S'05-M'10) was born in Oviedo, Spain, in 1980. He received the M.Sc. degree in electrical engineering from the University of Oviedo, Gijón, Spain, in 2005, and the Ph.D. degree from the same university in 2010.

Since February 2005, he has been a Researcher in the Department of Electrical and Electronic Engineering, University of Oviedo. Since February 2007, he has also been an Assistant Professor of electronics at the same university. His research interests include systems, and LED-based lighting. ac-dc and dc-dc converters, uninterruptible power

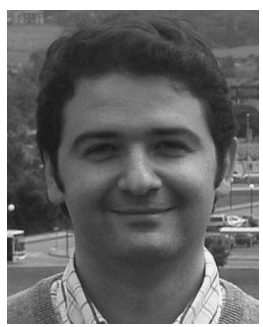

Diego G. Lamar (S'05-M'09) was born in Zaragoza, Spain, in 1974. He received the M.Sc. and $\mathrm{Ph} . \mathrm{D}$. degrees in electrical engineering from the Universidad de Oviedo, Gijón, Spain, in 2003 and 2008, respectively.

In 2003, he became a Research Engineer at the University of Oviedo, and since September 2005, he has been an Assistant Professor. His research interests are switching-mode power supplies, converter modeling, and power-factor-correction converters.

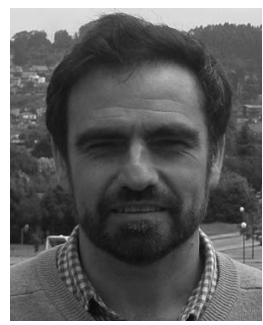

Javier Sebastián (M'87-SM'11) was born in Madrid, Spain, in 1958. He received the M.Sc. degree from the Polytechnic University of Madrid, Gijón, Madrid, in 1981, and the Ph.D. degree from the Universidad de Oviedo, Spain, in 1985.

He was an Assistant Professor and then an Associate Professor at both the Polytechnic University of Madrid and the Universidad de Oviedo. Since 1992, he has been with the Universidad de Oviedo, where he is currently a Professor. His research interests are switching-mode power supplies, modeling of dc-todc converters, low-output-voltage dc-to-dc converters, and high-power-factor rectifiers. 


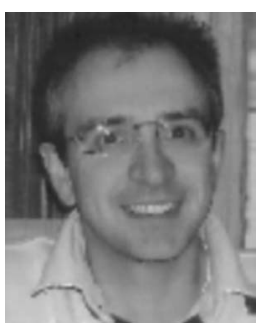

Didier Balocco received the Engineer diploma from the Ecole Nationale Supérieure d'Electronique et de Radio Electricité de Bordeaux, France, in 1992, and the Ph.D. degree in electrical engineering from the University of Bordeaux, Talence, France, in 1997.

In 1996, he joined Alcatel Converters (now AEG Power Solutions) as a Research Engineer. Since 2000, he has managed the research activities at AEG Power Solutions. He published more than ten papers on power electronics. He holds one patent, and three patents are pending. His main interests are switching-mode power supplies, converter stability, and high-power-factor rectifiers for telecom and industrial application. He has developed several projects for telecom domain and lighting.

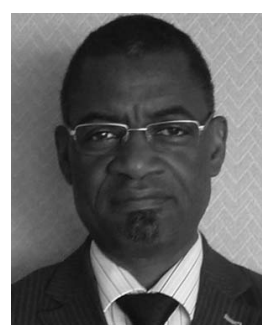

Almadidi Aguissa Diallo received the Engineer diploma from the Ecole Nationale d'Ingénieur de Bamako, Mali, in 1977, and the Specialization diploma of the Ecole Supérieure d'Electricité de Gif/Yvette, France, in 1979. In 1984, he received the Ph.D degree in electronic engineering from the University of Paris-Sud Orsay where he was an Assistant during several years.

In 1984, he joined Atelier d'Electronique et d'Automatisme, a company that later became Alcatel Converters (now AEG Power Solutions) as a Development Engineer, later becoming a Research Engineer. Since 2003, he has managed the operational marketing as a Product Line Manager with AEG Power Solutions. He has published a number of papers on power electronics. $\mathrm{He}$ holds three patents, and one patent is pending. His main interests are switchingmode power supplies, converter reliability, high-efficiency and high-powerfactor rectifiers for telecom and industrial application, and smart grid solutions. $\mathrm{He}$ has developed and introduced several projects for telecom domain, smart lighting, battery chargers, and smart grid. 